Final Report for the DoE grant "Photophysics of Two-dimensional Semiconductor Nanoparticle/Liquid Interfaces", grant number DE-FG03-01ER15222

Principle Investigator:

David F. Kelley

Departinent of Chemistry

Kansas State University

Manhattan KS 66506

\title{
Summary of Results:
}

The research supported by this grant has investigated the photophysics of two-dimensional semiconductor nanoparticles. The type of nanoparticles selected for study have fallen into two general categorics: the group VI B dichalcogenides $\left(M X_{2}\right.$, where $\left.M=M o, W ; X=S, S e\right)$ and the group III A chalcogenides (GaS, GaSe and InSe). The results obtained on each type of nanoparticle will be discussed below.

\section{MoS,-type nanoparticles:}

During the funding period of this grant we coinpleted the spectroscopic characterization of $\mathrm{MoS}_{2}$ and related types of nanoparticles. Most of the work was on $\mathrm{MoS}_{2}$, with additional studies on $\mathrm{MoSe}_{2}, \mathrm{WS}_{2}$ and $\mathrm{WeSe}_{2}$ nanoparticles. In order to establish the nature of the size-dependent electronic states, we first synthesized several different sizes of $\mathrm{MoS}_{2}$ nanoparticlcs. Each size was then characterized by TEM images, electron diffraction and optical spectroscopy. The layered structure of $\mathrm{MoS}_{2}$ results nanoparticles having a disc-like norphology. Electron diffraction and static spectroscopic results indicate that the particles are three atoms thick, specifically, single S-Mo-S trilayer discs. One interesting and very unusual result was that the different sizes of nanoparticles can be synthesized directly or may be synthesized from samples of polydisperse nanoparticles. This is not a size selection method, rather, the size distribution of the entire polydisperse sample is narrowed. The ability to do this indicates that certain nanoparticle sizes are themodynamically favored, that is, these sizes correspond to free energy minima. Thermodynamics are often found to play a role in the growth kinetics of nanoparticles. However, to our knowledge, this is the first observation of specific semiconductor nanoparticle sizes corresponding to thermodynamic local minima and thereby directly determining the nanoparticle size distribution. By changing the nature of the ligands in solution (and therefore bound to the nanoparticle surfaces) it is possible to change the surface free energy, and therefore the size corresponding to a free energy minimum. This situation is completely analogous to thermodynamic control of the size distribution of gold particles with adsorbed thiols. In the gold case, the results have been analyzed in terms of the surface versus bulk free energies and may be semiquantitatively understood by this thermodynamic model. Using the synthetic methods that we have developed, nanoparticles having diameters of $3.5,4.5$, and $8 \mathrm{~nm}$ are easily obtained. Static and time resolved absorption and emission spectroscopy was then used to determine the polarizations of several optical for each size of nanoparticle. Wc find that the cxtent of $z$-axis and $x . y$-plane quantum confinement varies with the size of the nanoparticle. Specifically, the energy 


\section{DISCLAIMER}

This report was prepared as an account of work sponsored by an agency of the United States Government. Neither the United States Government nor any agency Thereof, nor any of their employees, makes any warranty, express or implied, or assumes any legal liability or responsibility for the accuracy, completeness, or usefulness of any information, apparatus, product, or process disclosed, or represents that its use would not infringe privately owned rights. Reference herein to any specific commercial product, process, or service by trade name, trademark, manufacturer, or otherwise does not necessarily constitute or imply its endorsement, recommendation, or favoring by the United States Government or any agency thereof. The views and opinions of authors expressed herein do not necessarily state or reflect those of the United States Government or any agency thereof. 


\section{DISCLAIMER}

Portions of this document may be illegible in electronic image products. Images are produced from the best available original document. 
of z-polarized transitions varies much less strongly with nanoparticle diameter than does the energy of $x, y$-polarized transitions. As a result, the polarization of the lowest observed transition is size dependent and that the ordering of the lowest excited states changes as the particle size decreases. There is crossover between the lowest transition having $z$ - and $x, y$-polarization in 8 $\mathrm{nm}$ nanoparticles. Thus, the lowest observed transition in bulk $\mathrm{MoS}_{2}$ is $\mathrm{x}-\mathrm{y}$ polarized and we conclude that the ordering of this transition and the lowest $z$ polarized transition reverse for particles smaller than about $8 \mathrm{~nm}$. These assignments are summarized in the static absorption spectra, figure 1, below.

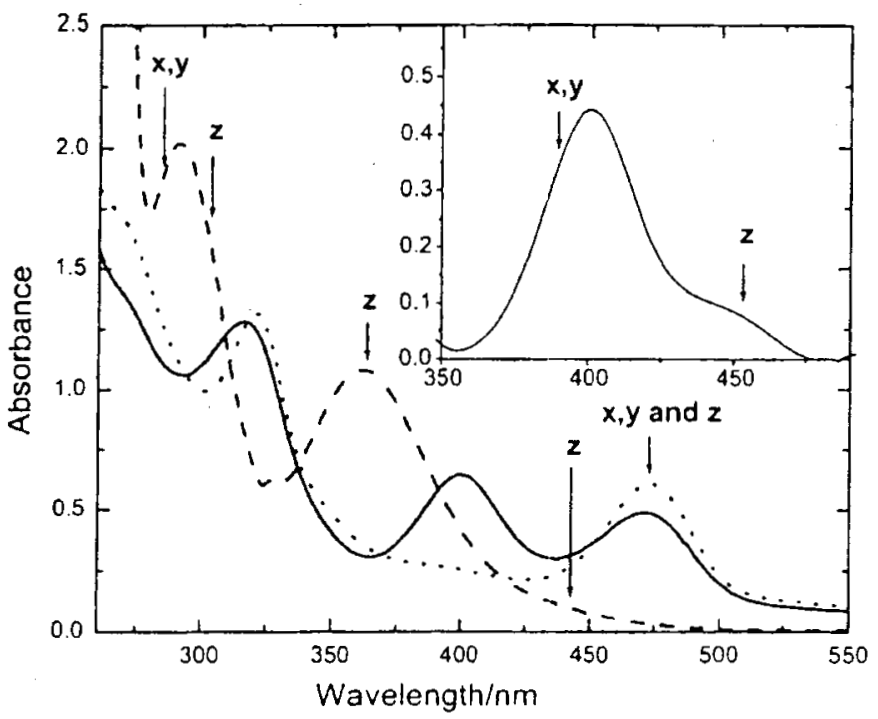

Figure 1. Absorption spectra of $3.5 \mathrm{~mm}$ (dashed curve). $8 \mathrm{~nm}$ (dotled curve) and of a mixture of 4.5 and 8 nm (solid curve) $\mathrm{MoS}_{2}$ nanoparticles. The mixlure curve corresponds to a $\mathrm{MoCl}_{4} /\left(\mathrm{NH}_{4}\right)_{2} \mathrm{~S}$ synthesis, shortly after injection. A difference curve corresponding to just the $4.5 \mathrm{~nm}$ nanoparticles is shown in the insert. The assigned polarizations of the absorption transitions atc also show'll.

\section{GaSe and InSe nanoparticles:}

During the previous DOE grant we developed the basic synthetic method to produce GaSe nanoparticles. During the present funding period we have further refined this synthesis and elucidated much of the spectroscopy and photophysics of these particles. We have also begun to apply similar techniques to InSe nanoparticles, although the development of this synthesis has just begun.

GaSe nanoparticles are produced by the reaction of trimethyl-gallium with trioctylphosphine selenium in a high temperature coordinating solvent, trioctyl-phosphine. We find that the size distributions of the particles nay be'focused by controlling the reactant concentrations. 
Monodisperse particles having sizes from $4 \mathrm{~nm}$ to $9 \mathrm{~nm}$ may be obtained. Alternatively, by controlling the reactant concentrations, polydisperse samples may obtained. These particles may be size segregated by column chromatography or size selective precipitation and a very wide range of relatively monodisperse nanoparticles are obtained. Electron diffraction results indicate that these particles have a two-dimensional single tetra-layer type structure. TEM images of 2.5 , 4 and $9 \mathrm{~nm}$ particles are show $\mathrm{n}$ in figure 2 , below.
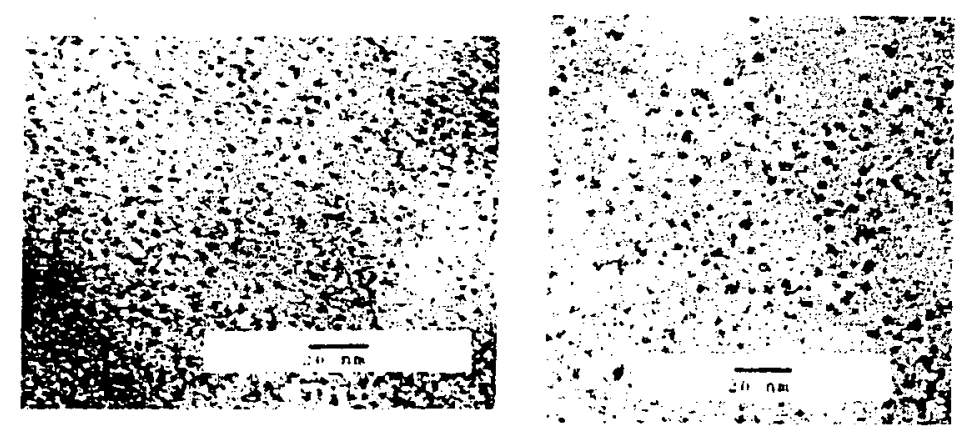

Figure 2 .

TEM image of Gase nanoparticles afier chromatographic purification (first fraction off of a silica get column). The particte diameters are $2.5+\% .5 \mathrm{~nm}$.

\begin{abstract}
Approximately $4 \mathrm{~nm}$ GaSe nanoparticles taken $90 \mathrm{~min}$ atter a single reactant injection. (This sample is used for chromatographic purification) The particle diameters range from 2 to $6 \mathrm{~nm}$.
\end{abstract}

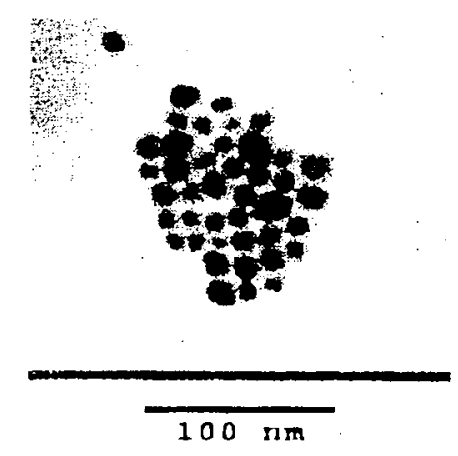

Larger $(8-10$ nim) GaSe nanoparticles. The particles size distribution is focused by multiple injections of the reactants.

The particles have absorption onsets in the 360 to $450 \mathrm{~nm}$ region, with the smallest particles absorbing furthest to the blue. The particles are cmissive, with emission quantum yields of about 10\%. Absorption and emission spectra of 2.5,4 and $9 \mathrm{~nm}$ particles as well as approximately $7 \mathrm{~nm}$ diameter InSe nanoparticles are shown in figure 3 , below. The studies on


Figure 3. Absorption and emission spectra of GaSe (lefi) and InSe (right) nanoparticles. The GaSe nanoparticles have sizes of $2.5 \mathrm{~mm}$ (left curve), $4 \mathrm{~nm}$ (center curves) and $9 \mathrm{~nm}$ (right curves). The solid and dashed 4 nm curves are for more and less polydisperse samples, respectively. The InSe nanoparticles are about 7 nm in diameter. 
InSe nanoparticles have just begun, and this report will focus on the spectroscopy and dynamics of GaSe nanoparticles. The photophysics and relaxation dynamics of GaSe nanoparticles have been studied in room temperature solutions, using static and time-resolved emission spectroscopy. The emission spectrum depends on the nanoparticles size, as shown in the figure above. This emission is polarized and the anisotropy is largest on the blue edge of the emission spectrum. Both the total (unpolarized) emission kinetics and the emission anisotropy kinetics are obtained. Static emission spectra along with wavelength dependent kinetic results permit the reconstruction of time dependent spectra. The kinetic results show an 80 ps decay component in the total enission, but not in the anisotropy decay kinetics. There is a ca. $270 \mathrm{~cm}^{-1}$ shift in the emission maximum during this decay. This transient is assigned to direct to indirect band edge relaxation. This is followed by a 400 ps decay in both the total emission and the anisotropy kinctics. A very small shift (ca. $170 \mathrm{~cm}^{-1}$ ) in the emission maximum occurs during this decay. This transient is assigned to trapping of holes in shallow acceptor levels. Finally, there is a $2.4 \mathrm{~ns}$ decay of the both the total emission and the emission anisotropy. As this decay occurs, the 480 nm emission is replaced by a much weaker, broad and unpolarized 520 nm enission. This decay is assigned to relaxation of holes into deep traps. The kinetic results are interpreted in terms of a model which is based on the relative energetics of the direct and indirect band edges and the hole trap states in bulk GaSe. This model is summarized in figure 4, below.

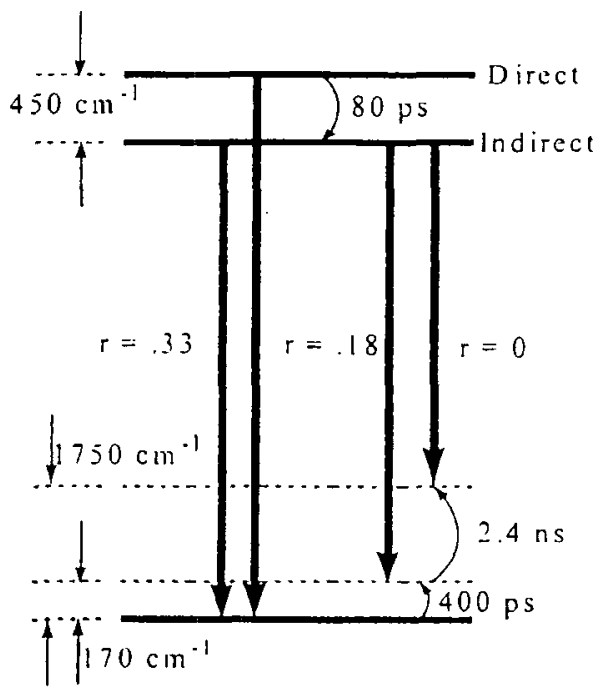

Figure 4. Summary of the relaxalion processes and times in GaSe nanoparticles. The anisotropies of the various emission processes are also indicated.

Most recently, we have studied the electron and hole intraband spectroscopy in GaSe nanoparticles having diameters ranging from 4 to $9 \mathrm{~nm}$. The assignment of the observed spectral features is facilitated by the use of femtosecond transient absorption polarization methods. The absorption transient are shown in figures 5 and 6 , below. The results indicate that the transient absorption spectrum in the 500 to $700 \mathrm{~nm}$ region has a size-independent peak at about $600 \mathrm{~nm}$. Polarization results indicate that the absorption anisotropy is small or negative on the blue edge of the spectrum, and increases with increasing wavelength. The anisotropy reaches a maximum 
in the $600 \mathrm{~nm}$ region, and remains approximately constant out to $700 \mathrm{~nm}$. Smaller particles exhibit smaller (or more negative) anisotropies and a larger wavelength dependence than larger particles.

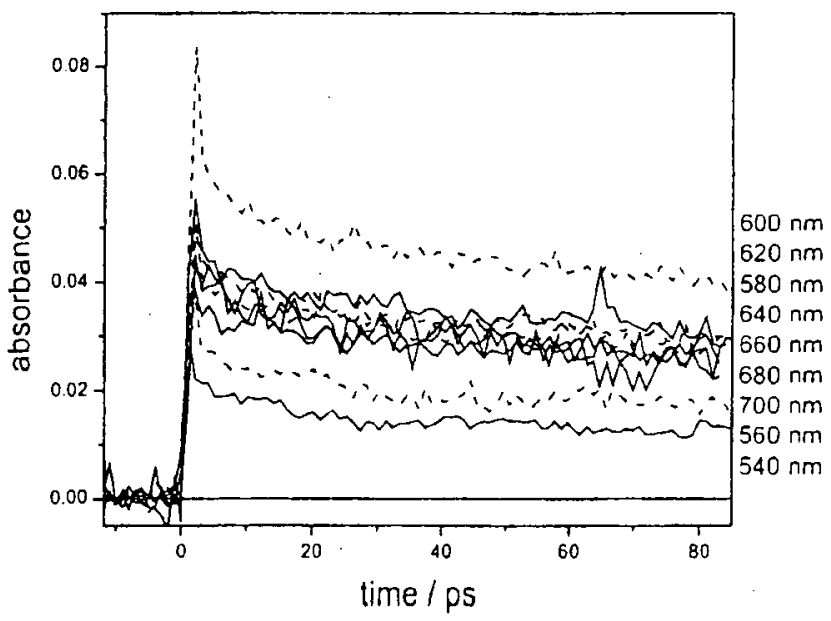

Figure 5. Time resolved absorption results oblained at different probe wavelenglhs. The transient in the first few picoseconds is due to a four wave mixing process, and is not of interest here.
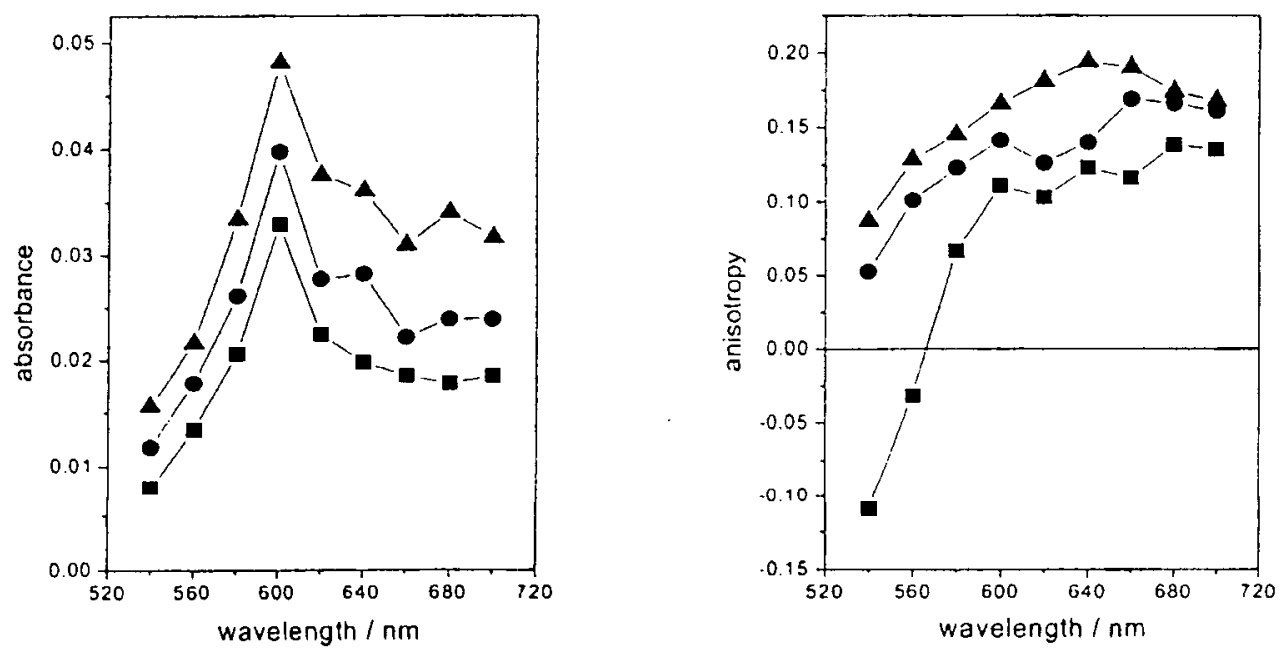

Figure 6. Absopption intensities and anisotropies of different sizes of GaSe nanoparticles obtained 10-30 ps following photoexcitation. Squares - $4 \mathrm{~nm}$. circles $-6 \mathrm{~nm}$. triangles $-8 \mathrm{~nm}$.

These results are interpreted in terms of a simple effective mass model. Due to the approximately cylindrical symmetry of these two-dimensional particles, the electron and hole states are described by particle-in-a-cylinder wavefunctions and the optical transitions may be calculated from these wavefunctions. Pyridine quenching studies indicate that the absorption is 
dominated by hole intraband transitions. The quantum confined hole levels and the intraband transitions are depicted in figure 7 . These calculations semiquantitatively predict the absorption
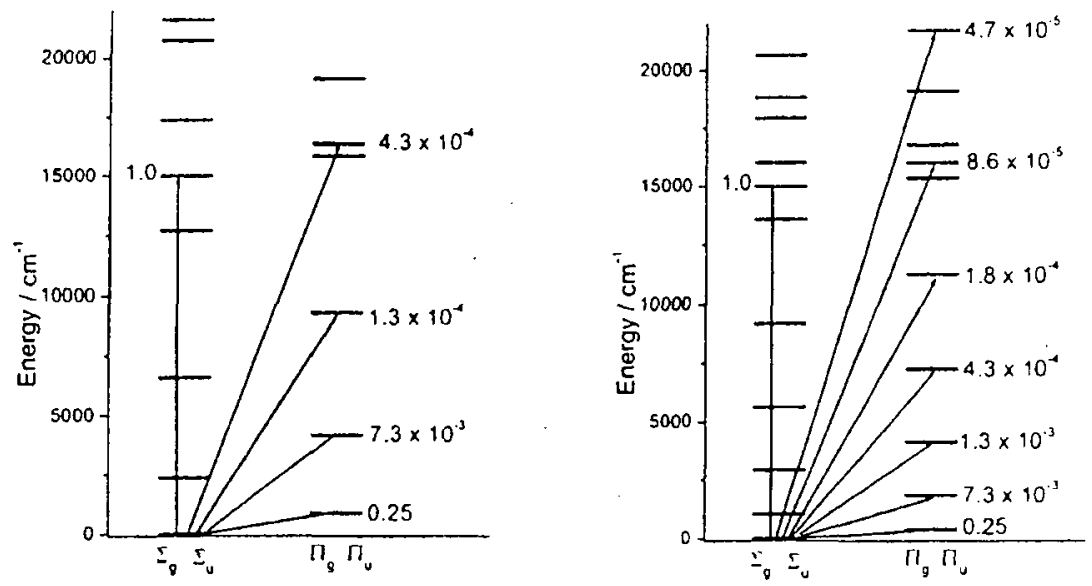

Figure 7. Calculated hole cnergies and intraband transition oscillalor strengths for $4 \mathrm{~nm}$ (left) and $6 \mathrm{~nm}$ (right) GaSe nanoparticles. "g" and " $u$ " states are indicated in black and gray, respectively.

maximum at $600 \mathrm{~nm}$ and qualitatively predict the wavelength dependence of the absorption polarization. The absolption peak at $600 \mathrm{~nm}$ corresponds the $\Sigma \rightarrow \Sigma$ hole transition, calculated at $15000 \mathrm{~cm}^{-1}(667 \mathrm{~nm})$. The calculations are also consistent with the size dependence of the anisotropy spectrum. Absorption near the $600 \mathrm{~nm}$ maximum is assigned to an out-of-plane hole intraband transition, while the transient absorptions to the blue of the maximum are assigned to in-plane electron and hole intraband transitions. The observation of a positive anisotropy at the longest wavelengths suggests the presence of a weak underlying, z-polarized electron intraband transition at redder wavelengths.

Papers acknowledging DoE support:

M. R. Waterland and D. F. Kelley, "Photophysics and Relaxation Dynamics of $\mathrm{Ru}(4,4 \text { 'dicarboxy-2,2'-bipyridine) })_{2}$ cis(NCS) 2 in Solution" J. Phys. Chem. A, 105, 4019 (2001).

V. Chikan and D. F. Kelley, "Size Dependent Spectroscopy of MoS 2 Nanoparticles" J. Phys. Chem. B-106, 3794 (2002).

V. Chikan and D. F. Kelley, "Synthesis of Highly Luminescent GaSe Nanoparticles" Nano Letters, 2, 141 (2002).

C. M. Olsen, M. R. Waterland and D. F. Kelley "Electron Injection Dynamics of N3-dye on Zirconia" J. Phys. Chem. B, 106, 6211 (2002):

D. F. Kelley "Nanoparticles of Layered Semiconductors" in Molecular and Supramolecular Phorochenistry. Vol. 10, Semiconductor Pholochemistry and Phorophysics, K. S. Schanze and V. Ramamurthy, eds., Marcel Dekker, New York, 173, (2003). 
V. Chikan and D. F. Kelley, "Relaxation Dynamics in Photoexcited GaSe Nanoparticles", $J$. Chem. Phys, 117, 8944 (2002).

V. Chikan and D. F. Kelley, "Spectroscopy, Dynamics and Electron Transfer in GaSe Nanoparticles", Proc. Soc. Photo-Optic. Inst. Engin., 4807, 36 (2002).

V. Chikan and D. F. Kelley, "Carrier Relaxation Dynamics in GaSe Nanoparticles", Nano Letters, 2, 1015 (2002).

H. Tu, V. Chikan and D. F. Kelley, "Electron and Hole Intraband Spectroscopy of GaSe Nanoparticles" J. Phys. Chem B. - in press 